\title{
Breaking of Lorentz invariance in electron-proton parity violation
}

\author{
Alexander Michel๑* and Marc Sher ${ }^{\dagger}$ \\ Physics Department, William \& Mary, Williamsburg, Virginia 23187, USA
}

(Received 28 September 2019; published 11 November 2019)

\begin{abstract}
A popular framework for exploring Lorentz violation is the Standard Model extension. This extension contains a large number of parameters that can be bounded in various experiments. Most studies, however, focus on the fermion or photon sector. Here, we consider Lorentz violation in the weak vector boson sector. The strongest bounds come from measurements of the asymmetry in Møller scattering. We study the bounds that can be obtained from future measurements of the parity violating asymmetry in deep inelastic electron-proton scattering at the EIC, the $\mathrm{LHeC}$, and the FCC-eh. For the FCC-eh, the bounds could be substantially improved over current bounds by including timing information.
\end{abstract}

DOI: 10.1103/PhysRevD.100.095011

\section{INTRODUCTION}

While the Standard Model is successful at describing physical phenomena at the highest energies measured, it is expected to break down when gravitational effects are no longer negligible. Since many models of quantum gravity are nonlocal, one might expect Lorentz invariance to be violated at a high energy scale. While it is expected that this high energy scale would be the Planck scale, requiring very high precision studies, larger effects are possible, and the question of Lorentz violation is one that should be probed experimentally.

In general, it is difficult to write the most general Lorentz violating theory. Even the meaning of a Lagrangian becomes uncertain in such a theory. An extremely useful approach was developed years ago by Colladay and Kostelecký [1-4], who constructed the Standard Model extension (SME). This model is based on the Standard Model, but adds Lorentz violating terms which satisfy the Standard Model gauge symmetry and have dimension less than or equal to four. The extra terms also are invariant under observer Lorentz transformations; i.e., all Lorentz indices must be contracted and the physics does not depend on the choice of coordinates. The Lorentz violation is also independent of position and time, so that energy and momentum are conserved. The model contains a large number of parameters that can be experimentally constrained, and there are many hundreds of papers studying

\footnotetext{
asmichel@email.wm.edu

†mtsher@wm.edu
}

Published by the American Physical Society under the terms of the Creative Commons Attribution 4.0 International license. Further distribution of this work must maintain attribution to the author(s) and the published article's title, journal citation, and DOI. Funded by SCOAP . constraints on these parameters (an extensive list, updated to 2016, can be found in Ref. [5]).

The vast majority of these studies have involved long-lived particles, and only a few have involved, for example, heavy scalar and vector bosons [6-16]. Most of these involve fermions, studying, for example, beta decays. Recently, $\mathrm{Fu}$ and Lehnert [17] considered bounds on parameters that only involve gauge bosons. They studied bounds involving internal $Z$ boson lines in electron-electron scattering experiments searching for parity violation and found that bounds from the E158 experiment at SLAC improved previous bounds by 2 orders of magnitude. The parameters they considered arise from the Lorentz violating terms in the SME

$$
\mathcal{L}=-\frac{1}{4}\left(k_{B}\right)_{\kappa \lambda \mu \nu} B^{\kappa \lambda} B^{\mu \nu}-\frac{1}{2}\left(k_{W}\right)_{\kappa \lambda \mu \nu} \operatorname{tr}\left(W^{\kappa \lambda} W^{\mu \nu}\right) .
$$

The coefficients $k_{B}$ and $k_{W}$ are real and dimensionless. They have the symmetries of the Riemann tensor and a vanishing double trace, so there are 19 coefficients each. These are $C P T$ even; we will not discuss $C P T$ odd terms since they are associated with negative energy contributions. Fu and Lehnert also discuss the fact that a relevant term in the Higgs kinetic energy SME Lagrangian will not have an effect on the results, and we will ignore that here. Writing the above Lagrangian term in terms of the photon and $Z$ gives

$$
\begin{aligned}
\mathcal{L}= & -\frac{1}{4}\left(k_{B} \cos ^{2} \theta_{W}+k_{W} \sin ^{2} \theta_{W}\right)_{\kappa \lambda \mu \nu} F^{\kappa \lambda} F^{\mu \nu} \\
& -\frac{1}{4}\left(k_{W} \cos ^{2} \theta_{W}+k_{B} \sin ^{2} \theta_{W}\right)_{\kappa \lambda \mu \nu} Z^{\kappa \lambda} Z^{\mu \nu} \\
& -\frac{1}{4} \sin 2 \theta_{W}\left(k_{W}-k_{B}\right)_{\kappa \lambda \mu \nu} F^{\kappa \lambda} Z^{\mu \nu} .
\end{aligned}
$$

Here, $F^{\mu \nu}=\partial^{\mu} A^{\nu}-\partial^{\nu} A^{\mu}, Z^{\mu \nu}=\partial^{\mu} Z^{\nu}-\partial^{\nu} Z^{\mu}$. The first term in this expression deals with Lorentz violation in the 


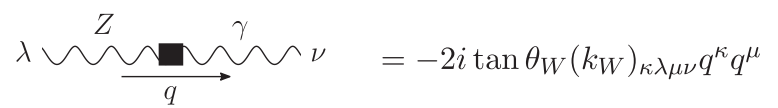

(a)

$\lambda \sim^{Z} \sim \sim_{q}^{Z} \sim \nu=-2 i\left(1-\tan ^{2} \theta_{W}\right)\left(k_{W}\right)_{\kappa \lambda \mu \nu} q^{\kappa} q^{\mu}$

(b)

FIG. 1. Propagator insertions afforded by Eq. (2).

QED sector. This is very strongly constrained by many experiments and is completely negligible, so one can set $k_{B}=-\tan ^{2} \theta_{W} k_{W}$. The resulting Feynman rules are given in Fig. 1. It is the $k_{W}$ coefficients that Fu and Lehnert bound from considerations of parity violation in electron-electron scattering. In this paper, we extend the work of $\mathrm{Fu}$ and Lehnert to include parity violation in electron-proton scattering. This has been measured precisely at Qweak, and will also be measured at the electron-ion collider (EIC) [18-20], Large Hadron-electron Collider (LHeC) [21], and eventually at the Future Circular Collider (FCC-eh) [22]. There have been studies of the effects of Lorentz violation in deep inelastic scattering [23-25], but these all consider the quark sector coefficients on the scattering. We considered the pure gauge sector coefficients. The model and calculations are presented in Sec. II and the results are discussed (for each proposed future experiment) in Sec. III, as are our conclusions.

\section{ELECTRON-PROTON SCATTERING}

In parity violation in elastic $e p$ scattering, such as at Qweak, the Lorentz violating effects are proportional to $Q^{2} / M_{Z}^{2}$, which is very small. This suppression makes it impossible to strengthen existing bounds on $k_{W}$.

We instead consider inelastic $e^{-} p \rightarrow e^{-} X$ scattering. Only energies high enough for the parton model to hold are considered. The $e^{-} p \rightarrow e^{-} X$ cross section in terms of the $e^{-} q_{i} \rightarrow e^{-} q_{i}$ quark subprocess cross section is

$$
\left(\frac{d^{2} \sigma}{d E^{\prime} d \Omega}\right)_{e p \rightarrow e X}=\sum_{i} \int_{0}^{1} d x f_{i}(x)\left(\frac{d^{2} \sigma}{d E^{\prime} d \Omega}\right)_{e q_{i} \rightarrow e q_{i}} .
$$

The functions $f_{i}(x)$ are the usual parton distribution functions (PDFs) and the sum over $i$ ranges over all quark flavors. The diagrams contributing to the quark subprocess are in Fig. 2.

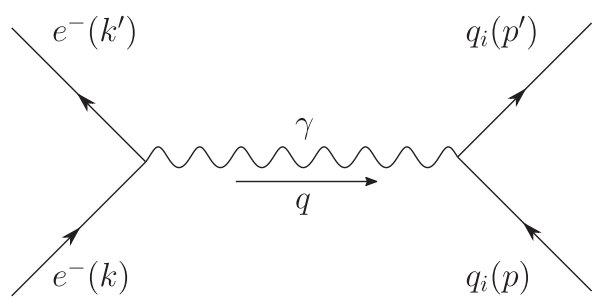

(a) $i \mathcal{M}_{\gamma}$

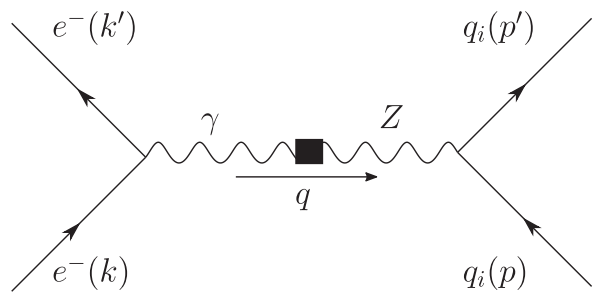

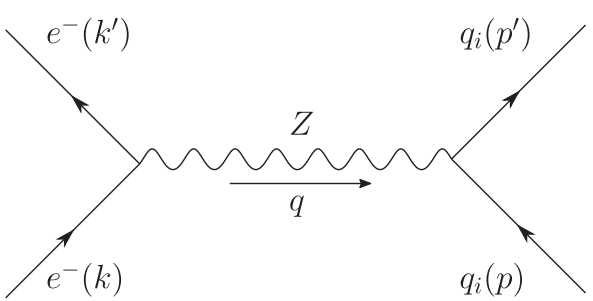

(b) $i \mathcal{M}_{Z}$

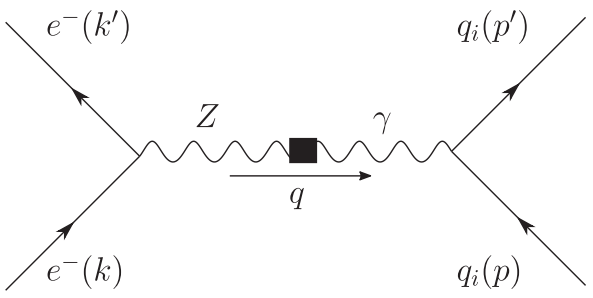

(d) $i \mathcal{M}_{Z \gamma}$

(c) $i \mathcal{M}_{\gamma Z}$



(e) $i \mathcal{M}_{Z Z}$

FIG. 2. Diagrams contributing to the process $e^{-} q_{i} \rightarrow e^{-} q_{i}$. 
We denote by $\left|\mathcal{M}_{i, h}\right|^{2}$ the squared sum of the diagrams in Fig. 2, where $h=L, R$ is the helicity of the incoming electron and $i$ is the quark flavor. This matrix element can be written

$$
\left|\mathcal{M}_{i, h}\right|^{2}=\left|\mathcal{M}_{i, h}\right|_{0}^{2}+\delta\left|\mathcal{M}_{i, h}\right|^{2}
$$

where $\left|\mathcal{M}_{i, h}\right|_{0}^{2}$ contains the usual standard model contribution and $\delta\left|\mathcal{M}_{i, h}\right|^{2}$ is the Lorentz violating correction. The explicit form of $\delta\left|\mathcal{M}_{i, h}\right|^{2}$ is rather long, so we have placed it in Appendix.

The primary observable is the asymmetry

$$
\mathcal{A} \equiv \frac{d \sigma_{L}(e p \rightarrow e X)-d \sigma_{R}(e p \rightarrow e X)}{d \sigma_{L}(e p \rightarrow e X)+d \sigma_{R}(e p \rightarrow e X)} .
$$

Expanded to leading order in $k_{W}$ in the lab frame, the asymmetry is of the form $\mathcal{A}=\mathcal{A}_{0}+\delta \mathcal{A}$ where $\mathcal{A}_{0}$ is the Standard Model result and $\delta \mathcal{A}$ is the Lorentz violating correction proportional to $k_{W}$.

In order to cast $\delta \mathcal{A}$ into a form suitable for numerical analysis, we employ a parametrization of $k_{W}$ due to $\mathrm{Fu}$ and Lehnert [17] in terms of a dimensionless vector $\boldsymbol{\xi}=\left(\xi_{x}, \xi_{y}, \xi_{z}\right)$. This is a substantial simplification of the analytical calculation, which reduces the 19-dimensional parameter space to a 3-dimensional parameter space. It seems reasonable, since time and space coordinates do not mix, but there is no general argument as to why these three parameters should dominate the effects. Fu and Lehnert then define $\xi^{\mu} \equiv(0, \boldsymbol{\xi})^{\mu}$ and $\zeta^{\mu} \equiv(1, \mathbf{0})^{\mu}$, and we can write

$$
\begin{aligned}
\left(k_{W}\right)^{\kappa \lambda \mu \nu}= & \frac{1}{2}\left[g^{\kappa \mu} \xi^{\{\lambda} \zeta^{\nu\}}-g^{\kappa \nu} \xi^{\left\{\lambda \zeta^{\mu\}}\right.}\right. \\
& \left.+g^{\lambda \nu} \xi^{\{\kappa} \zeta^{\mu\}}-g^{\lambda \mu} \xi^{\alpha \kappa} \zeta^{\nu\}}\right], \\
\text { where } \xi^{\{\mu} \zeta^{\nu\}} \equiv & \frac{1}{2}\left(\xi^{\mu} \zeta^{\nu}+\xi^{\nu} \zeta^{\mu}\right) .
\end{aligned}
$$

This parametrization reduces our problem to the bounding of $\xi$, which now expresses the entirety of the Lorentz violation effect.

One needs to consider the coordinate system in order to define $k_{W}$ (and hence $\boldsymbol{\xi}$ ). The canonical choice is suncentered celestial equatorial coordinates [3]. Consequently, the calculation of $\delta \mathcal{A}$ will require all momenta to be expressed in this frame. To change to these coordinates from those usually employed in scattering calculations ${ }^{1}$ one must first transform to the coordinate system with $\hat{\mathbf{x}}$ pointing south, $\hat{\mathbf{y}}$ pointing east, and $\hat{\mathbf{z}}$ normal to the Earth's surface. Changing to this reference frame will involve rotations that depend on the azimuthal scattering angle $\phi$ and the angle $\beta$ of the electron beam east of south. The rotation

\footnotetext{
${ }^{1}$ For example, coordinates chosen so that the plane of interaction is spanned by $\hat{\mathbf{z}}$ and either $\hat{\mathbf{x}}$ or $\hat{\mathbf{y}}$.
}

TABLE I. Explicit forms of the parameters in Eq. (7). Recall that $\beta$ is the beam angle east of south, $\chi$ is the colatitude of the beam, and $\theta(\phi)$ is the polar (azimuthal) scattering angle of the scattered electron.

\begin{tabular}{ll}
\hline \hline$\gamma$ & $\arctan (\tan \theta \sin \phi)$ \\
$\delta_{1}$ & $-\arctan (\tan \beta / \cos \chi)$ \\
$\delta_{2}$ & $-\arctan (\tan (\beta-\gamma) / \cos \chi)$ \\
$f_{1}$ & $\sqrt{1-\sin ^{2} \beta \cos ^{2} \chi}$ \\
$f_{2}$ & $\sqrt{1-\sin ^{2} \theta \cos ^{2} \phi} \sqrt{1-\sin ^{2}(\beta-\gamma) \cos ^{2} \chi}$ \\
$f_{3}$ & $|\sin \theta \cos \phi \sin \chi|$ \\
\hline \hline
\end{tabular}

$$
\left(\begin{array}{ccc}
\cos \chi \cos \Omega_{\oplus} t & -\sin \Omega_{\oplus} t & \sin \chi \cos \Omega_{\oplus} t \\
\cos \chi \sin \Omega_{\oplus} t & \cos \Omega_{\oplus} t & \sin \chi \sin \Omega_{\oplus} t \\
-\sin \chi & 0 & \cos \chi
\end{array}\right)
$$

then transforms to the sun-centered celestial equatorial coordinate system [3]. Here, $\Omega_{\oplus}=2 \pi /(23 \mathrm{~h} 56 \mathrm{~min})$ and $\chi$ is the colatitude of the lab. This last rotation need not include a boost, since the Earth's motion is completely nonrelativistic.

Notice that the only 3 -vectors appearing in the problem are the incoming (outgoing) electron momenta $\mathbf{k}\left(\mathbf{k}^{\prime}\right)$ and the parton momentum $\mathbf{p}$. Then since $\delta \mathcal{A}$ is a scalar quantity proportional to $k_{W}$, it must be a linear combination of the dot products $\hat{\mathbf{k}} \cdot \boldsymbol{\xi}, \hat{\mathbf{k}}^{\prime} \cdot \boldsymbol{\xi}$, and $\hat{\mathbf{p}} \cdot \boldsymbol{\xi}$. It is then straightforward to show that

$$
\begin{aligned}
\hat{\mathbf{p}} \cdot \boldsymbol{\xi} & =-\hat{\mathbf{k}} \cdot \boldsymbol{\xi} \\
\hat{\mathbf{k}} \cdot \boldsymbol{\xi} & =f_{1} \cos \left(\Omega_{\oplus} t-\delta_{1}\right) \sqrt{\xi_{x}^{2}+\xi_{y}^{2}}+\mathrm{const} \\
\hat{\mathbf{k}}^{\prime} \cdot \boldsymbol{\xi} & =\left(f_{2} \cos \left(\Omega_{\oplus} t-\delta_{2}\right)+f_{3} \cos \Omega_{\oplus} t\right) \sqrt{\xi_{x}^{2}+\xi_{y}^{2}}+\text { const. }
\end{aligned}
$$

The parameters used in the above expressions are given in Table I. The overall additive constants we have chosen to omit above are time independent and therefore not easily disentangled from Standard Model effects. Fu and Lehnert also find a dependence on $\sqrt{\xi_{x}^{2}+\xi_{y}^{2}}$. The $\xi_{z}$ piece is not sensitive to the time dependence since the $z$ direction is perpendicular to the Earth's surface, and the azimuthal dependence vanishes, as they show, in the ultrarelativistic limit. Using Eq. (7) we can cast the correction into its final form

$\delta \mathcal{A}=A(\theta, \phi) \cos \left(\Omega_{\oplus} t-\delta(\theta, \phi)\right) \sqrt{\xi_{x}^{2}+\xi_{y}^{2}}+$ const.

The computation of $\delta$ and $A$ must be done numerically as a function of $\theta$, all the parameters in Table I, and the beam 




(a) Fixed $\beta=45^{\circ}$

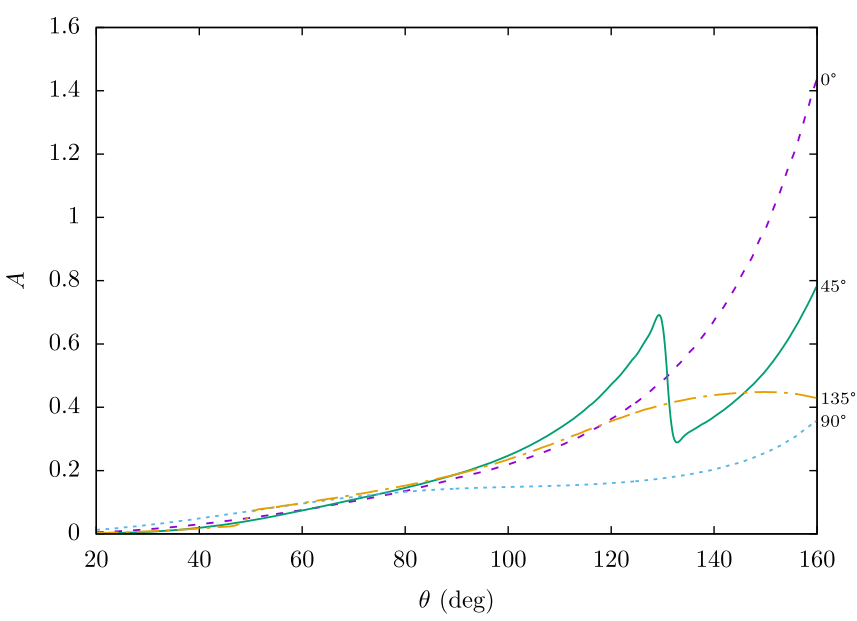

(b) Fixed $\phi=60^{\circ}$

FIG. 3. Graphs of $A(\theta, \phi)$ for FCC-eh energies. In (a), $\beta$ is fixed to $45^{\circ}$ while $\phi$ takes on the values on the right of the graph. The reverse is true for (b).

energies associated with the given experiment. This is the subject of the next section.

\section{RESULTS AND CONCLUSIONS}

The calculation is now straightforward. One uses Eq. (A3), with $F$ (the proton energy) replaced by the quark energy, $x F$, and then kinematically replaces $E^{\prime}$ with

$$
\frac{2 x E F}{E+x F-(E-x F) \cos \theta} \text {. }
$$

The $c_{i}^{V, A}$ are then input into Eq. (A2), and then the matrix elements in Eq. (A4) are summed. Note the factor of $h$ in the matrix elements - this is -1 for left-handed electrons and +1 for right-handed electrons. The dot products involving $\xi$ are in Eq. (7). Then one integrates over the parton distributions and the asymmetry is determined. The final form is in Eq. (8), and we just need to determine $A(\theta, \phi)$.

The resulting asymmetry depends on the colatitude, $\chi$, which we will take to be $45 \mathrm{deg}$ (this is fairly close to all current and future laboratories). It depends on $\beta$, the angle of the beam east of south, which will, in due course, be known for any collider. The only remaining variables are the polar and azimuthal angles of the scattered electrons. One could, of course, integrate over these variables, but it is useful to see the dependence explicitly.

Our results for the FCC-eh are in Fig. 3. In Fig. 3(a), we show the value of $A$ [in Eq. (8)] as a function of $\theta$ for various values of $\phi$. Here, we have set $\beta=45 \mathrm{deg}$. Note that the Lorentz violating corrections are peaked in the backward direction. In Fig. 3(b), we show the value of $A$ as a function of $\theta$ for various values of $\beta$, setting $\phi=60 \mathrm{deg}$. If one integrates over the solid angle in the backward hemisphere and divides by $2 \pi$, one can get an "average" value of $A$. Typical values of $A$ are $0.3-0.7$, depending on $\beta$, and thus the result in Eq. (8) is this value times $\sqrt{\xi_{x}^{2}+\xi_{y}^{2}}$ times an oscillation with a period of the Earth's rotational period.

In Fig. 4, we have given the results for the $\mathrm{LHeC}$. The general structure is similar to the FCC-eh, but the amplitude is roughly an order of magnitude smaller. We will not show results for the EIC-the structure is similar but the amplitude is 2-3 orders of magnitude smaller. The reason that the LHeC and EIC have much smaller effects is entirely due to the center of mass energy of the colliders. The LHeC has a proton beam that has an order of magnitude less energy, and the EIC has an even smaller energy.

In the work of $\mathrm{Fu}$ and Lehnert [17], the value of $\sqrt{\xi_{x}^{2}+\xi_{y}^{2}}$ was bounded by studying the E158 experiment at SLAC [26,27], which studied Møller scattering. The bound arose by requiring that the amplitude of the fluctuation be smaller than the statistical uncertainty in the E158 experiment, and gave an upper bound of $3.4 \times 10^{-7}$. They noted that the upcoming MOLLER experiment at Jefferson Lab would, using the same argument, be able to improve this by a factor of 1.4. They also pointed out that the different beam directions might provide access to different components of $\left(k_{W}\right)_{\kappa \lambda \mu \nu}$; thus even if the bound could not be improved, it would be valuable to search for Lorentz violation. This is already a low bound, and it might seem difficult for the FCC-eh to do much better.

The Fu-Lehnert analysis only considered the amplitude of the oscillation - the actual rotation period of the Earth did not enter into the analysis. This is not surprising, since the timing of events for E158 are not available. If such timing is included for the FCC-eh, then it might be possible to perform a fit including the explicit time dependence and thus improving the bound. Even if one does not do this, it is 


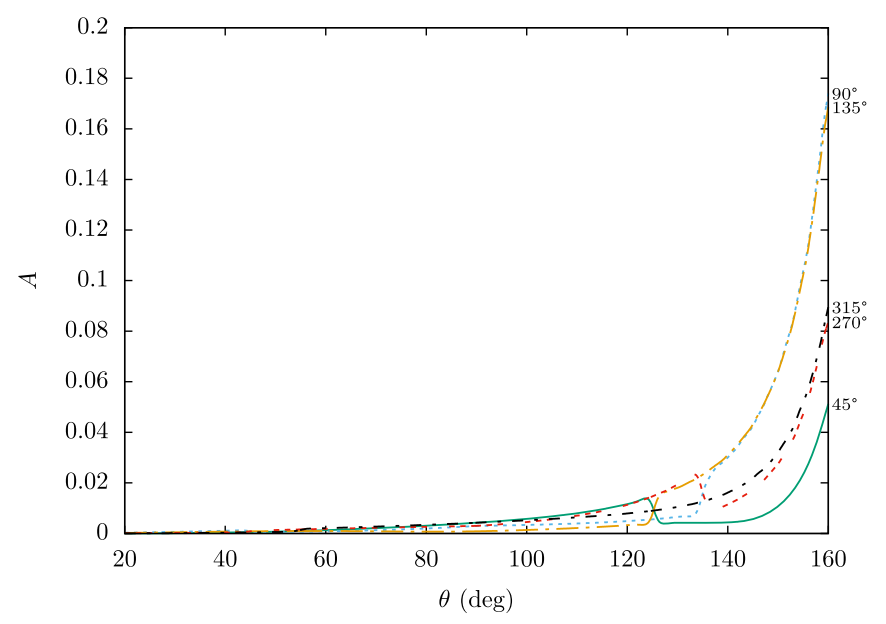

(a) Fixed $\beta=45^{\circ}$

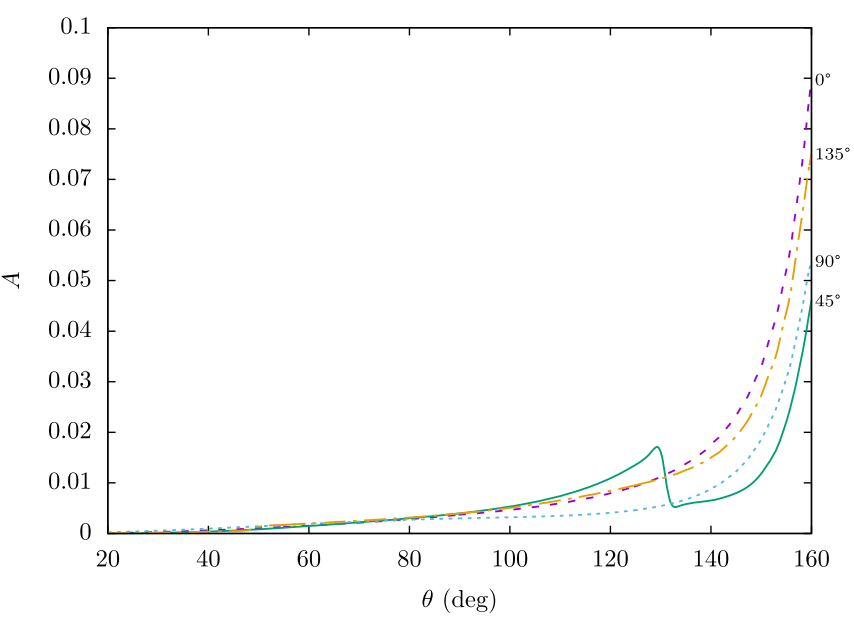

(b) Fixed $\phi=60^{\circ}$

FIG. 4. Graphs of $A(\theta, \phi)$ for $\mathrm{LHeC}$ energies.

likely that a good bound could be obtained. While we do not know the estimated uncertainties in deep-inelastic scattering (DIS) parity violation at the FCC-eh, it has been reported [22] that DIS at the FCC-eh will be sensitive to $\sin ^{2} \theta_{W}$ to an accuracy of 0.001 . This is a factor of 4 better than the E158 experimental uncertainty, and thus it is plausible that the FCC-eh will improve the bound substantially. At the $\mathrm{LHeC}$, the size of the effect is an order of magnitude smaller, but a comparable bound might still be reached.

Even if the bound obtained by E158 cannot be reached, one must remember that the Fu-Lehnert parametrization of Eq. (5), while quite reasonable, essentially considers a three-dimensional slice of the 19-dimensional space of $\left(k_{W}\right)_{\kappa \lambda \mu \nu}$. Thus, a fit including a potential time dependence in future deep inelastic experiments could prove worthwhile.

In future deep inelastic parity violation experiments, the values of the latitude and electron beam direction will be fixed, and it will be straightforward to use our results to find the polar and azimuthal angular dependence of the Lorentz violating effect. By fitting to a sinusoidal dependence with a period of the Earth's rotation, one will be able to bound $\sqrt{\xi_{x}^{2}+\xi_{y}^{2}}$, if the Fu-Lehnert parametrization is adopted. But even without that parametrization, a search would be worthwhile, since it is sensitive to the entire 19dimensional space of $\left(k_{W}\right)_{\kappa \lambda \mu \nu}$.

\section{ACKNOWLEDGMENTS}

We are grateful to Wouter Deconinck, David Armstrong, Jianwei Qiu, Michael Kordosky, and Chris Carone for useful discussions. We thank the National Science Foundation for support under Grant No. PHY-1819575. This work was performed in part using computing facilities at the College of William and Mary that were provided by contributions from the National Science Foundation, the Commonwealth of Virginia Equipment Trust Fund, and the Office of Naval Research.

\section{APPENDIX: COMPUTATION OF $\left.\delta|| \mathcal{M}_{i, h}\right|^{2}$}

The terms in

$$
\begin{aligned}
\delta\left|\mathcal{M}_{i, h}\right|^{2}= & 2 \Re\left\{\mathcal{M}_{\gamma}^{*} \mathcal{M}_{\gamma Z}+\mathcal{M}_{\gamma}^{*} \mathcal{M}_{Z \gamma}+\mathcal{M}_{\gamma}^{*} \mathcal{M}_{Z Z}\right. \\
& \left.+\mathcal{M}_{Z}^{*} \mathcal{M}_{\gamma Z}+\mathcal{M}_{Z}^{*} \mathcal{M}_{Z \gamma}+\mathcal{M}_{Z}^{*} \mathcal{M}_{Z Z}\right\}
\end{aligned}
$$

all have the same trace structure. Each has a factor of $\left(k_{W}\right)^{\kappa \lambda \mu \nu}\left(k_{\kappa}^{\prime}-k_{\kappa}\right)\left(k_{\mu}^{\prime}-k_{\mu}\right)$ times either of

$\operatorname{tr}\left[k / \gamma^{\lambda} \not k \gamma_{\rho}\right] \operatorname{tr}\left[\not p / \gamma^{\nu} \not p \gamma^{\rho}\right] \quad$ or $\operatorname{tr}\left[\gamma^{5} \not k \gamma^{\lambda} \mid k \gamma_{\rho}\right] \operatorname{tr}\left[\gamma^{5} \not p / \gamma^{\nu} \not \not \gamma^{\rho}\right]$.

Let $E$ be the incoming electron energy, $E^{\prime}$ the scattered electron energy, $\theta$ the scattering angle, and $F$ the incoming proton energy. Then using the Fu-Lehnert parametrization one can write $\left(k_{W}\right)^{\kappa \lambda \mu \nu}\left(k_{\kappa}^{\prime}-k_{\kappa}\right)\left(k_{\mu}^{\prime}-k_{\mu}\right)$ times either of the traces in Eq. (A1) as

$$
Y^{V, A}=c_{1}^{V, A} \mathbf{k} \cdot \boldsymbol{\xi}+c_{2}^{V, A} \mathbf{k}^{\prime} \cdot \boldsymbol{\xi}+c_{3}^{V, A} \mathbf{p} \cdot \boldsymbol{\xi}
$$

Here, $c_{j}^{V}$ and $c_{j}^{A}$ refer to the first and second traces in Eq. (A1), respectively. Abbreviating $c \equiv \cos \theta$ for notational clarity, one finds 


$$
\begin{aligned}
& c_{1}^{V}=8 F E^{\prime}\left[-5\left(1-c^{2}\right) E E^{\prime} F+4(c-1) E^{3}-2(1+c) E^{2}\left((c-1) E^{\prime}-F\right)+(c+1)^{2} E^{\prime 2} F\right], \\
& c_{2}^{V}=16 E F\left[(c-1) E E^{\prime}\left(2 E^{\prime}+F\right)-(c+1) E^{\prime 2}\left((c-1) E^{\prime}+(c-2) F\right)-2 E^{2} F\right], \\
& c_{3}^{V}=8(c-1) E E^{\prime} F\left[(7 c+5) E E^{\prime}-(c+1) E^{\prime 2}-2 E^{2},\right. \\
& c_{1}^{A}=8(1-c) E E^{\prime} F\left[(1+c) E E^{\prime}+(1+c) E^{\prime 2}-4 E^{2}\right], \\
& c_{2}^{A}=16(1-c) E E^{\prime} F\left(E-E^{\prime}\right)\left((1+c) E^{\prime}+E\right), \\
& c_{3}^{A}=-8(1-c)^{2} E^{2} E^{\prime 2}\left(E+E^{\prime}\right) .
\end{aligned}
$$

This gives

$$
\begin{aligned}
2 \Re\left(M_{\gamma}^{*} M_{\gamma Z}\right) & =\frac{-Q e^{4}}{2 \cos ^{2} \theta_{w} t^{2}\left(t-M_{Z}^{2}\right)}\left(G_{V} Y^{V}-h G_{A} Y^{A}\right), \\
2 \Re\left(M_{\gamma}^{*} M_{Z \gamma}\right) & =\frac{Q^{2} e^{4}\left(g_{V}-h g_{A}\right)}{2 \cos ^{2} \theta_{w} t^{2}\left(t-M_{Z}^{2}\right)} Y^{V}, \\
2 \Re\left(M_{\gamma}^{*} M_{Z Z}\right) & =\frac{Q e^{4}\left(1-\tan \theta_{W}\right)\left(g_{V}-h g_{A}\right)}{4 \cos ^{2} \theta_{w} \sin ^{2} \theta_{w} t\left(t-M_{Z}^{2}\right)^{2}}\left(G_{V} Y^{V}-h G_{A} Y^{A}\right), \\
2 \Re\left(M_{Z}^{*} M_{\gamma Z}\right) & =\frac{\left(g_{V}-h G_{A}\right) e^{4}}{8 \cos ^{4} \theta_{w} \sin ^{2} \theta_{w} t\left(t-M_{Z}^{2}\right)}\left(\left(G_{V}^{2}+G_{A}^{2}\right) Y^{V}-2 G_{V} G_{A} h Y^{A}\right), \\
2 \Re\left(M_{Z}^{*} M_{Z \gamma}\right) & =\frac{-\left(g_{V}-h g_{A}\right)^{2} Q e^{4}}{8 \sin ^{2} \theta_{w} \cos ^{4} \theta_{w} t\left(t-M_{Z}^{2}\right)^{2}}\left(G_{V} Y^{V}-h G_{A} Y^{A}\right), \\
2 \Re\left(M_{Z}^{*} M_{Z Z}\right) & =\frac{-\left(g_{V}-h g_{A}\right)^{2} e^{4}\left(1-\tan \theta_{w}\right)}{16 \sin ^{4} \theta_{w} \cos ^{4} \theta_{w}\left(t-M_{Z}^{2}\right)^{3}}\left(\left(G_{V}^{2}+G_{A}^{2}\right) Y^{V}-2 G_{V} G_{A} h Y^{A}\right) .
\end{aligned}
$$

Here, $g_{V}$ and $g_{A}\left(G_{V}\right.$ and $\left.G_{A}\right)$ are the vector and axial vector couplings of the electron and quarks, respectively, and $Q$ is the quark charge.

[1] D. Colladay and V. A. Kostelecký, Phys. Rev. D 55, 6760 (1997).

[2] D. Colladay and V. A. Kostelecký, Phys. Rev. D 58, 116002 (1998).

[3] V. A. Kostelecky and M. Mewes, Phys. Rev. D 66, 056005 (2002).

[4] V. A. Kostelecký, Phys. Rev. D 69, 105009 (2004).

[5] V. A. Kostelecký and N. Russell, Rev. Mod. Phys. 83, 11 (2011).

[6] B. Altschul, Astropart. Phys. 28, 380 (2007).

[7] D. L. Anderson, M. Sher, and I. Turan, Phys. Rev. D 70, 016001 (2004).

[8] E. O. Iltan, Eur. Phys. J. C 40, 269 (2005).

[9] E. O. Iltan, Mod. Phys. Lett. A 19, 327 (2004).

[10] E. O. Iltan, Acta Phys. Pol. B 36, 2955 (2005).

[11] J. I. Aranda, F. Ramírez-Zavaleta, D. A. Rosete, F. J. Tlachino, J. J. Toscano, and E. S. Tututi, J. Phys. G 41, 055003 (2014).

[12] J. I. Aranda, F. Ramírez-Zavaleta, F. J. Tlachino, J. J. Toscano, and E. S. Tututi, Int. J. Mod. Phys. A 29, 1450180 (2014).

[13] J. P. Noordmans, H. W. Wilschut, and R. G. E. Timmermans, Phys. Rev. C 87, 055502 (2013).
[14] J. P. Noordmans, C. J. G. Onderwater, H. W. Wilschut, and R. G. E. Timmermans, Phys. Rev. D 93, 116001 (2016).

[15] E. A. Dijck et al., Ann. Phys. (Amsterdam) 525, 653 (2013).

[16] K. K. Vos, H. W. Wilschut, and R. G. E. Timmermans, Phys. Rev. C 92, 052501 (2015).

[17] H. Fu and R. Lehnert, Phys. Lett. B 762, 33 (2016).

[18] A. Accardi et al., Eur. Phys. J. A 52, 268 (2016).

[19] E. C. Aschenauer et al., arXiv:1409.1633.

[20] S. Abeyratne et al., arXiv:1504.07961.

[21] J. L. Abelleira Fernandez et al. (LHeC Study Group), J. Phys. G 39, 075001 (2012).

[22] A. Abada et al. (FCC Collaboration), Eur. Phys. J. C 79, 474 (2019).

[23] V. A. Kostelecky, E. Lunghi, and A. R. Vieira, Phys. Lett. B 769, 272 (2017).

[24] E. Lunghi and N. Sherrill, Phys. Rev. D 98, 115018 (2018).

[25] V. A. Kostelecky and Z. Li, Phys. Rev. D 99, 056016 (2019).

[26] P. L. Anthony et al. (SLAC E158 Collaboration), Phys. Rev. Lett. 92, 181602 (2004).

[27] P. L. Anthony et al. (SLAC E158 Collaboration), Phys. Rev. Lett. 95, 081601 (2005). 